University of Wollongong

Research Online

Faculty of Engineering and Information

Faculty of Engineering and Information

Sciences - Papers: Part A

Sciences

$1-1-2012$

Double trigonal warping and the anomalous quantum Hall step in bilayer graphene with Rashba spin-orbit coupling

Bo Wang

Peking University

Chao Zhang

University of Wollongong, czhang@uow.edu.au

Zhongshui Ma

Peking University, zma@uow.edu.au

Follow this and additional works at: https://ro.uow.edu.au/eispapers

Research Online is the open access institutional repository for the University of Wollongong. For further information contact the UOW Library: research-pubs@uow.edu.au 


\title{
Double trigonal warping and the anomalous quantum Hall step in bilayer graphene with Rashba spin-orbit coupling
}

\author{
Abstract \\ We demonstrate that the trigonal warping observed in bilayer graphene is doubled in the presence of \\ Rashba spin-orbit (RSO) coupling, i.e.the Dirac points along the three-fold symmetry axis are doubled. \\ There are now seven Dirac points. Furthermore, the RSO interaction breaks the electron-hole symmetry of \\ the magnetic band structure. The most intriguing feature is that the step of the quantum Hall plateau at \\ zero energy is four times that at finite energy. The number of Dirac points and the zero energy Hall step \\ are only determined by the existence of RSO coupling, but are independent of the strength of the coupling. \\ The robustness of these phenomena suggests equivalence between the RSO coupling and the topological \\ effect in bilayer coupling. (c) 2012 IOP Publishing Ltd.
}

\section{Keywords}

orbit, quantum, hall, step, coupling, bilayer, graphene, rashba, spin, trigonal, warping, double, anomalous

\section{Publication Details}

Wang, B., Zhang, C. \& Ma, Z. (2012). Double trigonal warping and the anomalous quantum Hall step in bilayer graphene with Rashba spin-orbit coupling. Journal of Physics: Condensed Matter, 24 (48), 1-8. 
Double trigonal warping and the anomalous quantum Hall step in bilayer graphene with Rashba spin-orbit coupling

This article has been downloaded from IOPscience. Please scroll down to see the full text article.

2012 J. Phys.: Condens. Matter 24485303

(http://iopscience.iop.org/0953-8984/24/48/485303)

View the table of contents for this issue, or go to the journal homepage for more

Download details:

IP Address: 130.130.37.84

The article was downloaded on 03/12/2012 at 04:35

Please note that terms and conditions apply. 


\title{
Double trigonal warping and the anomalous quantum Hall step in bilayer graphene with Rashba spin-orbit coupling
}

\author{
Bo Wang ${ }^{1}$, C Zhang ${ }^{2}$ and Zhongshui Ma ${ }^{1}$ \\ ${ }^{1}$ School of Physics, Peking University, Beijing 100871, People's Republic of China \\ ${ }^{2}$ School of Physics, University of Wollongong, New South Wales 2522, Australia
}

Received 6 September 2012, in final form 19 October 2012

Published 7 November 2012

Online at stacks.iop.org/JPhysCM/24/485303

\begin{abstract}
We demonstrate that the trigonal warping observed in bilayer graphene is doubled in the presence of Rashba spin-orbit (RSO) coupling, i.e. the Dirac points along the three-fold symmetry axis are doubled. There are now seven Dirac points. Furthermore, the RSO interaction breaks the electron-hole symmetry of the magnetic band structure. The most intriguing feature is that the step of the quantum Hall plateau at zero energy is four times that at finite energy. The number of Dirac points and the zero energy Hall step are only determined by the existence of RSO coupling, but are independent of the strength of the coupling. The robustness of these phenomena suggests equivalence between the RSO coupling and the topological effect in bilayer coupling.
\end{abstract}

(Some figures may appear in colour only in the online journal)

\section{Introduction}

Since the isolation of single layers of graphite in 2004 [1], a lot of exciting work on single layer graphene (SLG) has been carried out [2], for example the prediction and observation of electron-hole symmetry and a half-integer quantum Hall effect [3-5], finite conductivity at zero charge-carrier concentration [3], the strong suppression of weak localization [6-8], universal conductance [9-11], magnetic enhancement of the optical conductance in graphene nanoribbons [12] and a strong nonlinear response in the terahertz frequency regime $[13,14]$.

More recently, attention has also been paid to SLG's cousin, bilayer graphene (BLG). Electrons in bilayers can exhibit qualitatively new properties not seen in single layers, such as interlayer drags [15] and correlations [16]. The electronic and transport properties of BLG differ significantly from SLG in many respects, particularly at low energies in the 'Dirac' regime. The electronic band structure of bilayer graphene has an asymmetry gap [17]. In bilayer graphene the semiconductor gap can be controlled experimentally [18]. The optical and magneto-optical far infrared properties of bilayer graphene have been studied [19]. The role of the impurities in biased bilayer graphene has been clarified [20]. One feature unique to BLG is the trigonal warping in the low-energy dispersion. The minimum conductivity in BLG is six times as large as that for single layer graphene [21]. This six times enhancement is independent of the strength of the trigonal warping, indicating the topological nature of the bilayer coupling.

In this paper, we present theoretical results on the role of Rashba spin-orbit (RSO) coupling on the electronic and transport properties of BLG. It is shown that the interplay of the RSO interaction (which lifts the spin degeneracy) and the interlayer couplings (which result in trigonal warping) can lead to an energy state whose dispersion exhibits double trigonal warping. Our main results are the following. (i) While the bilayer coupling changes the magnetic period from 1 to 6 , RSO coupling lifts the degeneracy and induces breakdown of the electron-hole symmetry near zero energy. (ii) The number of edge states increases to six. (iii) There is an anomalous Hall step at zero energy. We shall show the possible signature of trigonal warping imprinted on the edge state structure of zigzag graphene nanoribbons (ZGNRs). 


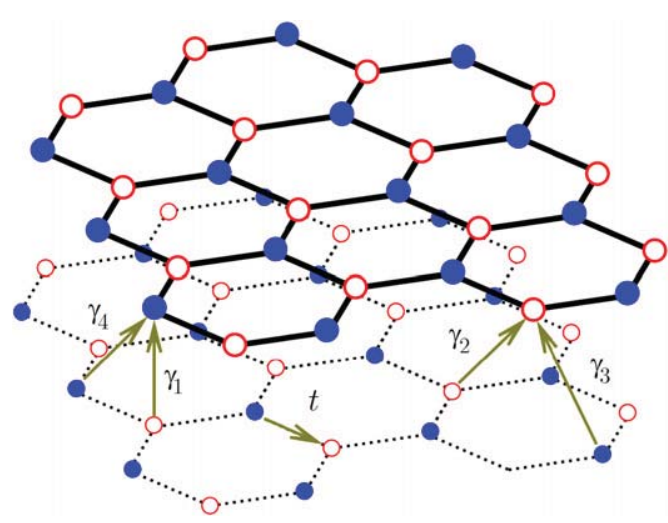

Figure 1. The bilayer graphene showing various coupling parameters: $t$ is for the nearest neighbor A-B and B-A coupling in SLG, $\gamma_{1}$ and $\gamma_{3}$ are for the nearest neighbor $A_{2}-B_{1}$ and $B_{2}-A_{1}$ coupling, and $\gamma_{2}$ and $\gamma_{4}$ are for $A_{2}-A_{1}$ and $B_{2}-B_{1}$ coupling in $B L G$.

The paper is organized as follows. In section 2 we present the formalism and result for the electronic properties of BLG with RSO coupling. Section 3 concerns the properties of BLG under a perpendicular magnetic field. Section 4 contains analysis and discussion on the transport properties. Concluding remarks are given in section 5 .

\section{Electronic properties}

A typical BLG sheet consists of two SLG layers stacked in the orientation shown in figure 1. The hollow and solid spheres represent atoms in the A- and B-sublattices. Several forms of the Hamiltonian for BLG are used in the literature depending on the approximations used and the relative orientations of the two layers. The original consideration was given by Slonczewski-Weiss-McClure, where all three interlayer coupling terms were included [21-23]. We consider that the two layers are arranged in the Bernal stacking $\left(\mathrm{A}_{2}-\mathrm{B}_{1}\right)$. The most prominent interlayer coupling terms are the $A_{2}-B_{1}$ and $\mathrm{B}_{2}-\mathrm{A}_{1}$ coupling between sites which are directly above (or below) each other [21, 24, 25]. Here we denote these terms as $\gamma_{1}=0.36 \mathrm{eV}$ for the $\mathrm{A}_{2}-\mathrm{B}_{1}$ coupling and $\gamma_{3}=0.315 \mathrm{eV}$ for the $\mathrm{B}_{2}-\mathrm{A}_{1}$ coupling. The other two interlayer coupling terms are the $A_{1}-A_{2}$ and $B_{1}-B_{2}$ coupling between inequivalent sites which are not directly above or below each other, but offset by an amount $b=1.42 \AA$. These two coupling terms have the same strength and are defined here as $t_{3}=t_{1}=0.12 \mathrm{eV}$. Finally, as usual, the nearest neighbor A-B and B-A coupling in the same layer is included, which is given here by $t=$ $3.16 \mathrm{eV}$. All energies will be normalized relative to the nearest neighbor hopping bandwidth $t$.

The Hamiltonian of BLG in the presence of RSO couplings can be written as

$$
\begin{aligned}
H= & t \sum_{\langle i, j\rangle} c_{i}^{\dagger} c_{j} \\
& +\sum_{l} t_{l} \sum_{\langle\alpha, \beta\rangle} c_{\alpha}^{\dagger} c_{\beta}+\mathrm{i} \lambda_{\mathrm{R}} \sum_{\langle i, j\rangle} c_{i}^{\dagger}\left(\boldsymbol{\sigma} \times \mathbf{d}_{i j}\right)_{z} c_{j},
\end{aligned}
$$

where $\mathbf{d}_{i j}$ is the displacement vector between the lattice sites $i$ and $j$ in a single graphene layer, $\boldsymbol{\sigma}$ are the Pauli matrices, $\gamma_{l}$ (defined above as $\gamma_{1}$ to $\gamma_{4}$ ) are the hopping energies between lattice sites $\alpha$ and $\beta$ in different graphene layers. The last term is the RSO coupling. It couples only nearest neighbors with opposite spin. In general, one also has intrinsic SO coupling [26]. The intrinsic SO interaction is generally much weaker (only a few $\mu \mathrm{eV}$ [27]) than the RSO coupling. Therefore we only include the RSO coupling in the Hamiltonian. The presence of the RSO coupling is due to the breaking of the inversion symmetry of the graphene lattice. The coefficient $\lambda_{\mathrm{R}}$ depends on the strength of the perpendicular electric field or the interaction with a substrate [28, 29]. Some papers have reported that the RSO can be tuned to $1 \mathrm{meV}$ for typical values of an external electric field $(50 \mathrm{~V} / 300 \mathrm{~nm})$. Breaking of the up-down symmetry by a substrate can result in an RSO coupling on the scale of $10 \mathrm{meV}$ [30]. The effect of impurities can increase the RSO coupling to $7 \mathrm{meV}$ [31]. Therefore, the strength of the RSO coupling is stronger than that of the intrinsic SO coupling by three orders of magnitude. For this reason, we shall focus our studies only on the effect of extrinsic SO coupling (which is also referred to as RSO coupling) [32]. In the rest of this paper, we shall use a dimensionless RSO coupling coefficient $\lambda=\lambda_{\mathrm{R}} a / t$.

We consider a four-band model (two in the conduction band and two in the valence band) and take into account the indices for pseudo-spin and real-spin. The eight-component wavefunction can be written as

$$
\begin{aligned}
& \left(\psi_{\mathrm{A}_{1}} \psi_{\mathrm{B}_{1}} \psi_{\mathrm{A}_{2}} \psi_{\mathrm{B}_{2}}\right)^{\mathrm{T}} \\
& \quad=\left(\psi_{\mathrm{A}_{1} \uparrow} \psi_{\mathrm{A}_{1} \downarrow} \psi_{\mathrm{B}_{1} \uparrow} \psi_{\mathrm{B}_{1} \downarrow} \psi_{\mathrm{A}_{2} \uparrow} \psi_{\mathrm{A}_{2} \downarrow} \psi_{\mathrm{B}_{2} \uparrow} \psi_{\mathrm{B}_{2} \downarrow},\right)^{\mathrm{T}},
\end{aligned}
$$

where $\mathrm{T}$ represents transpose, $\psi_{\mathrm{A}}$ and $\psi_{\mathrm{B}}$ are the wavefunctions of sublattices $\mathrm{A}$ and $\mathrm{B}$, and the index 1 (2) refers to the top (bottom) layer.

In the absence of RSO coupling, the translation invariance holds in both the $x$ and $y$ directions for infinite bilayer graphene. The wavefunction can be expressed in terms of Bloch wavefunctions. The Hamiltonian with RSO coupling in the basis of Bloch functions can be written as

$$
\widetilde{H}=\left(\begin{array}{cccc}
0 & t \chi+\lambda \eta & \gamma_{2} \chi & \gamma_{4} \chi^{*} \\
t \chi^{*}+\lambda \eta^{\dagger} & 0 & \gamma_{1} & \gamma_{4} \chi \\
\gamma_{2} \chi^{*} & \gamma_{1} & 0 & t \chi+\lambda \eta \\
\gamma_{3} \chi & \gamma_{4} \chi^{*} & t \chi^{*}+\lambda \eta^{\dagger} & 0
\end{array}\right),
$$

where

$$
\chi=\mathrm{e}^{\mathrm{i} k_{x} a}+2 \mathrm{e}^{-\mathrm{i} k_{x} \frac{a}{2}} \cos \left(\frac{\sqrt{3} a}{2} k_{y}\right)
$$

and

$$
\begin{aligned}
\eta= & -\sigma_{x} \sqrt{3} \sin \left(k_{y} \frac{\sqrt{3} a}{2}\right) \mathrm{e}^{-\mathrm{i} k_{x} \frac{a}{2}} \\
& -\mathrm{i} \sigma_{y}\left[\mathrm{e}^{\mathrm{i} k_{x} a}-\mathrm{e}^{-\mathrm{i} k_{x} \frac{a}{2}} \cos \left(k_{y} \frac{\sqrt{3} a}{2}\right)\right],
\end{aligned}
$$



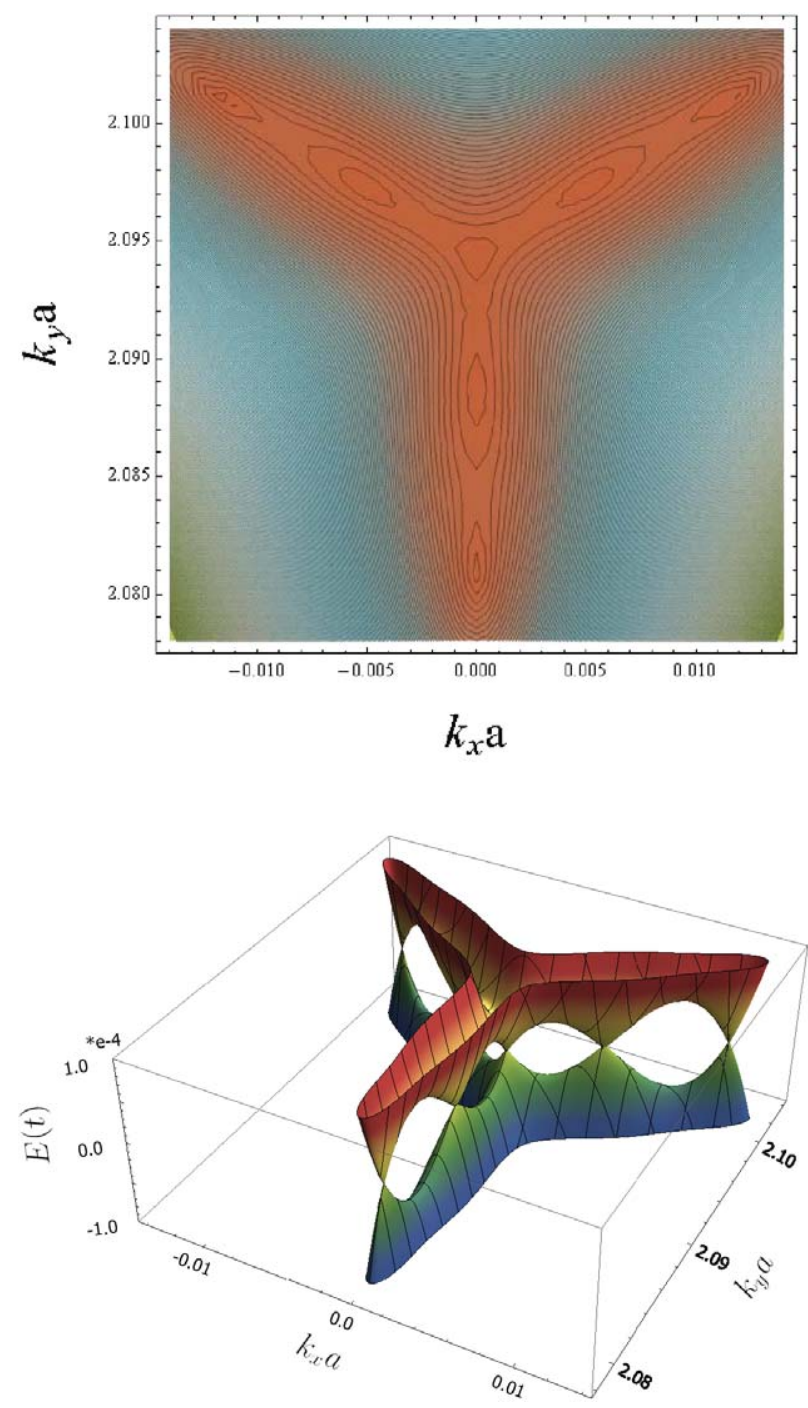

Figure 2. Top: the double trigonal warping at fixed energy. Bottom: a three dimensional view of the energy contour. The central point is $\left(0, \frac{2 \pi}{3}\right)$, which is a Dirac point in SLG. Every Dirac point in SLG expands to a double trigonal warping in BLG.

where $a$ is the $\mathrm{C}-\mathrm{C}$ bond length. By diagonalizing the Hamiltonian we can determine the eigenvalues. The energy contours are shown in figure 2. The interlayer coupling splits the original Dirac point, which gives rise to the well known trigonal warping. On the $k_{x}-k_{y}$ plane, there are four Dirac points, one at the K-point, and the other three at positions $\delta \mathbf{k}_{i}$ from the K-point with a $c_{3 v}$ symmetry, i.e., $\left|\mathbf{k}_{i}\right|$ is constant for $i=1-3$. The effect of the RSO interaction is to further split the three Dirac points at $\delta \mathbf{k}$. In total there are now seven identical points (split K-points), as shown in figure 2(a). One of them remains at the K-point which is unaffected by the RSO interaction. The ones at $\delta \mathbf{k}_{i}$ split into two Dirac points, located at $\mathbf{k}_{i a}$ and $\mathbf{k}_{i b}$. Figure 3(a) shows the cut of the energy contour at fixed $k_{x}$. The two new Dirac points are located on the two sides of the original Dirac point at $k_{i a}<k_{i}<k_{i b}$. The six other Dirac points form two triangles around the K-point. We refer to this energy contour as double trigonal warping (shown in figure 2(a)). Our results show quantitatively the
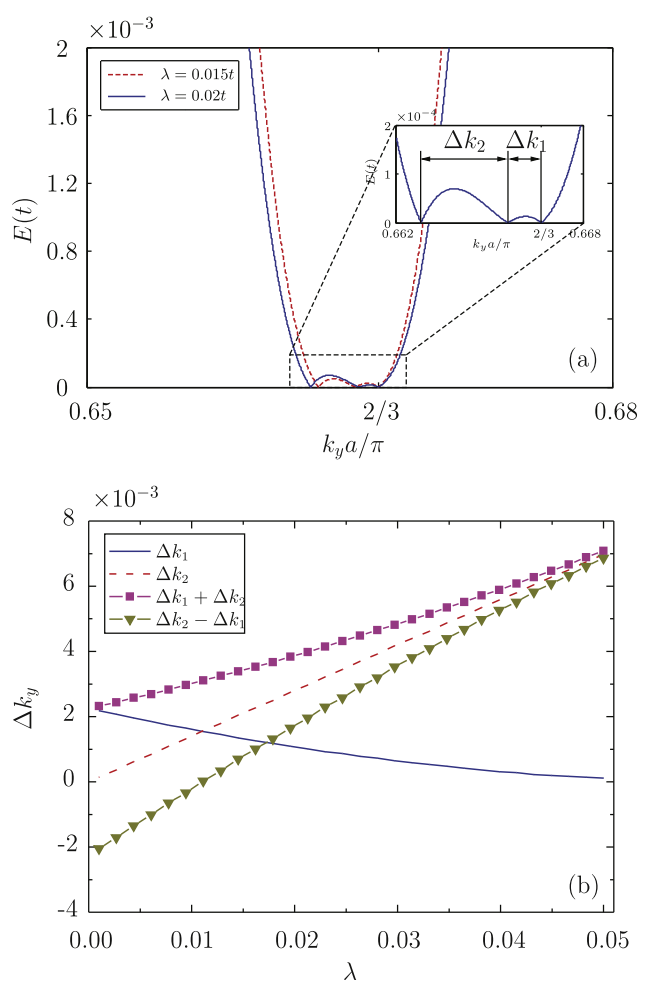

Figure 3. The influence of RSO coupling on the double trigonal warping. Top: the dispersion in the plane $k_{x}=0$ for two different RSO couplings. Bottom: the RSO-dependent distances between various Dirac points $\Delta k_{1}$ and $\Delta k_{2}$. The original Dirac point $\left(0, \frac{2 \pi}{3}\right)$ in SLG remains stationary in BLG.

equivalent roles played by RSO and interlayer coupling in trigonal warping. In the limit of the RSO coupling being much stronger than the interlayer coupling, the two Dirac points become very close. In this case there are three equivalent Dirac points at finite $\delta \mathbf{k}$. This is the case of single trigonal warping solely due to a large RSO interaction in single layer graphene. The distance between the two new Dirac points, $\Delta k_{2}=\mathbf{k}_{i a}-\mathbf{k}_{i b}$, is proportional to the strength of the RSO interaction. The distance between the K-point and the next Dirac point is $\delta k_{1}$, which decreases as the RSO coupling increases and approaches zero in the limit of large RSO coupling. Figure 3 depicts the dependence of the distance between various Dirac point on the strength of the RSO coupling $\lambda$. The distance $\delta k_{1}+\delta k_{2}$, which defines the region of double trigonal warping, increases almost linearly with the strength of RSO coupling. We note that the double trigonal warping induced by the RSO coupling occurs at very low energy and therefore cannot be calculated perturbatively.

\section{The effect of RSO interaction on the magnetic subbands}

The energy spectra of bilayer graphene in a magnetic field and with finite RSO coupling have been calculated recently [34, 35]. In the representation of equation (1), since the coupling only connects the nearest neighbors with opposite spins, the RSO interaction term under a finite magnetic field can be 
written in the form (see appendix)

$$
\begin{aligned}
H_{\mathrm{soc}} \psi_{A_{m}}= & -\sqrt{3} \lambda \sigma_{x} \sin \left[\pi f\left(m-\frac{1}{6}\right)+\frac{\sqrt{3}}{2} k_{y} a\right] \psi_{B_{m}} \\
& -\mathrm{i} \lambda \sigma_{y} \cos \left[\pi f\left(m-\frac{1}{6}\right)+\frac{\sqrt{3}}{2} k_{y} a\right] \psi_{B_{m}} \\
& -\mathrm{i} \lambda \sigma_{y} \psi_{B_{m+1}}
\end{aligned}
$$

and

$$
\begin{aligned}
H_{\mathrm{soc}} \psi_{B_{m}}= & -\sqrt{3} \lambda \sigma_{x} \sin \left[\pi f\left(m+\frac{1}{6}\right)+\frac{\sqrt{3}}{2} k_{y} a\right] \psi_{A_{m}} \\
& +i \lambda \sigma_{y} \cos \left[\pi f\left(m+\frac{1}{6}\right)+\frac{\sqrt{3}}{2} k_{y} a\right] \psi_{A_{m}} \\
& +i \lambda \sigma_{y} \psi_{A_{m+1}}
\end{aligned}
$$

where $f=\phi / \phi_{0} . \phi=3 \sqrt{3} B a^{2} / 2$ is the magnetic flux through the hexagon in the unit cell and $\phi_{0}=h c / e$ is the flux quantum. Due to the presence of a nonzero magnetic field, the Schrödinger equation becomes a Harper equation. It can be written as

$$
H \psi_{m}=\tilde{A}_{m} \psi_{m-1}+\tilde{C}_{m} \psi_{m}+\tilde{A}_{m}^{\dagger} \psi_{m+1},
$$

where

$$
\tilde{A}_{m}=\left(\begin{array}{cccc}
0 & \mathbf{0} & 0 & 0 \\
t \mathbf{I}_{2}+\mathrm{i} \lambda \sigma_{y} & 0 & 0 & 0 \\
\gamma_{2} \mathbf{I}_{2} & 0 & 0 & \mathbf{0} \\
2 \gamma_{3} \chi_{-\frac{1}{6}} \mathbf{I}_{2} & \gamma_{4} \mathbf{I}_{2} & t \mathbf{I}_{2}+\mathrm{i} \lambda \sigma_{y} & 0
\end{array}\right)
$$

and

$$
C_{m}=\left(\begin{array}{cccc}
0 & 2 t \chi_{\frac{1}{2}} \mathbf{I}_{2}-\lambda Y_{\frac{1}{2}}^{+} & 2 \gamma_{2} \chi_{\frac{1}{2}} \mathbf{I}_{2} & \gamma_{3} \mathbf{I}_{2} \\
2 t \chi_{\frac{1}{2}} \mathbf{I}_{2}-\lambda Y_{\frac{1}{2}}^{-} & 0 & \gamma_{1} \mathbf{I}_{2} & 2 \gamma_{4} \chi_{\frac{1}{6}} \mathbf{I}_{2} \\
2 \gamma_{2} \chi_{\frac{1}{2}} \mathbf{I}_{2} & \gamma_{1} \mathbf{I}_{2} & 0 & 2 t \chi_{\frac{1}{6}} \mathbf{I}_{2}-\lambda Y_{\frac{1}{6}}^{+} \\
\gamma_{3} \mathbf{I}_{2} & 2 \gamma_{4} \chi_{\frac{1}{6}} \mathbf{I}_{2} & 2 t \chi_{\frac{1}{6}} \mathbf{I}_{2}-\lambda Y_{\frac{1}{6}}^{-} & 0
\end{array}\right),
$$

where $\chi_{l}=\cos [\pi f(m+l)+\kappa], \tilde{\chi}_{l}=\sin [\pi f(m+l)$ $+\kappa], Y_{l}^{ \pm}=\sqrt{3} \sigma_{x} \tilde{\chi}_{l} \pm \mathrm{i} \sigma_{y} \chi_{l}$ and $\kappa=\frac{\sqrt{3} a}{2} k_{y}$.

In figure 4 we present the electronic energy versus the magnetic field over a full magnetic period. Compared to the spectrum of a single layer graphene sheet [33], the magnetic Brillouin zone has a six-fold increase. The reflection symmetry about $f=1 / 2$ is broken and the spectra are symmetric about $f=3$. The magnetic period of the spectra in $f$ is 6, i.e., $E(6+f)=E(f)$. The reason is that in bilayer graphene this is the extra magnetic phase shift of $\pi / 6$ (shown in equations (2) and (3)). We have previously shown that in single layer graphene when the next nearest neighbor hopping is included the magnetic period increases from 1 to 6 [36]. Including next nearest neighbor hopping in single layer graphene means that an electron couples to six additional electrons. In bilayer graphene, interlayer coupling also results in an electron coupling to six additional electrons in the other layer. Therefore the change of the magnetic period from 1 to 6 in bilayer graphene has the same origin as that in single layer

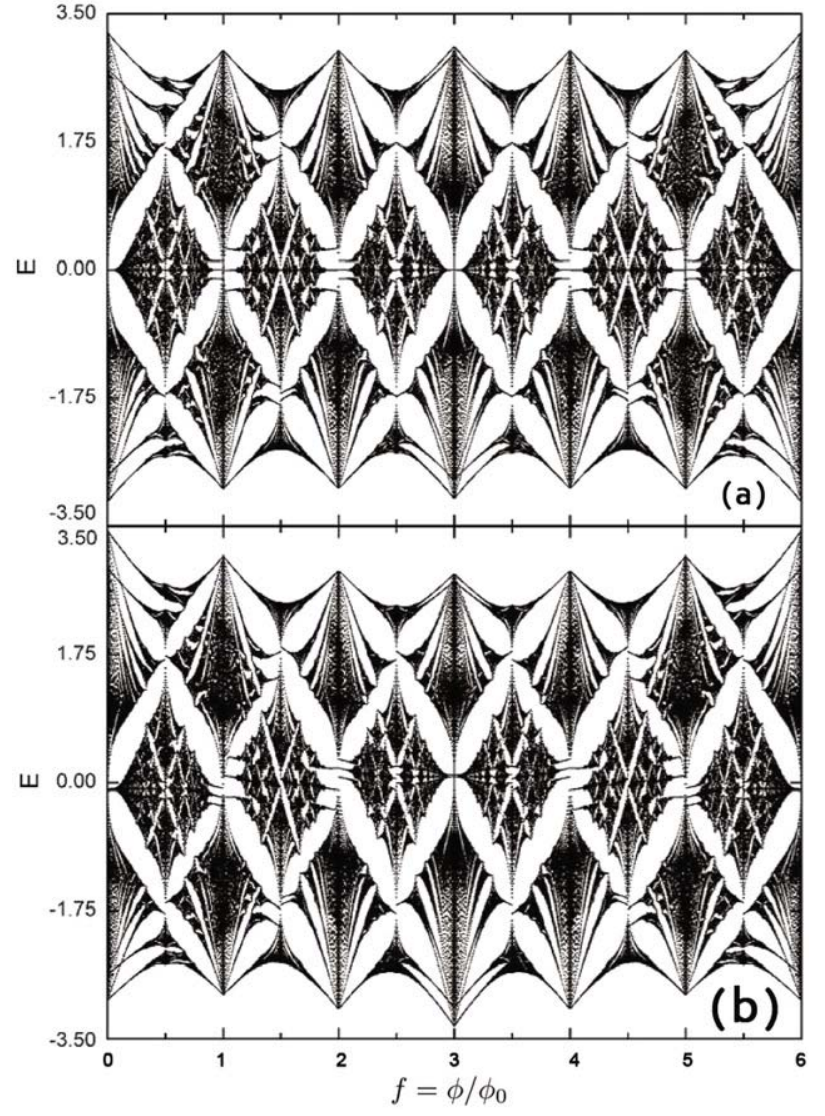

Figure 4. The energy spectrum of bilayer graphene in the presence of a magnetic field. (a) $\lambda=0$ and (b) $\lambda=0.1$.

graphene with next nearest neighbor hopping. The interlayer coupling does not break the electron-hole symmetry near zero energy. With the RSO coupling, the spectrum loses the zero energy electron-hole symmetry. The spectrum consists of $8 q$ subbands for a given $f=p / q$. For $f=0,6$, the RSO interaction induced level splitting at $E=0$ is clearly resolved. One of the interesting features in the magnetic subband arising from the RSO interaction is the level realigning at $f=1,2,4,5$. Without the RSO interaction, the $E=0$ level continues across these $f$ boundaries. With the finite RSO interaction, the level shift at these $f$ values is discrete. For example, the $E=0$ level on the left (or right) jumps to align with the first conduction (or valence) level on the right (or left). This jump in level alignment may provide a mechanism for quantum switching.

The constant magnetic field induces an electronic orbital magnetic moment. The magnetization per electron is defined as $M=-(1 / N)(\mathrm{d} F / \mathrm{d} B)$. In infinite BLG, the energy band structure shown in figure 4 leads to quantum magnetic oscillation in $M$. BLG ribbons are formed under an additional one-dimensional confinement. The quantization of the transverse momentum in the ribbon gives rise to additional quantum oscillations. In figure 5 we plot the magnetization curve as well as the susceptibility, $\chi=-(1 / N)\left(\mathrm{d}^{2} F / \mathrm{d} B^{2}\right)$, for two ribbons of different widths, as a function of the magnetic field. The $M-B$ curves for BLG with RSO coupling are shown in figure 5. The magnetization oscillates with the $B$-field 


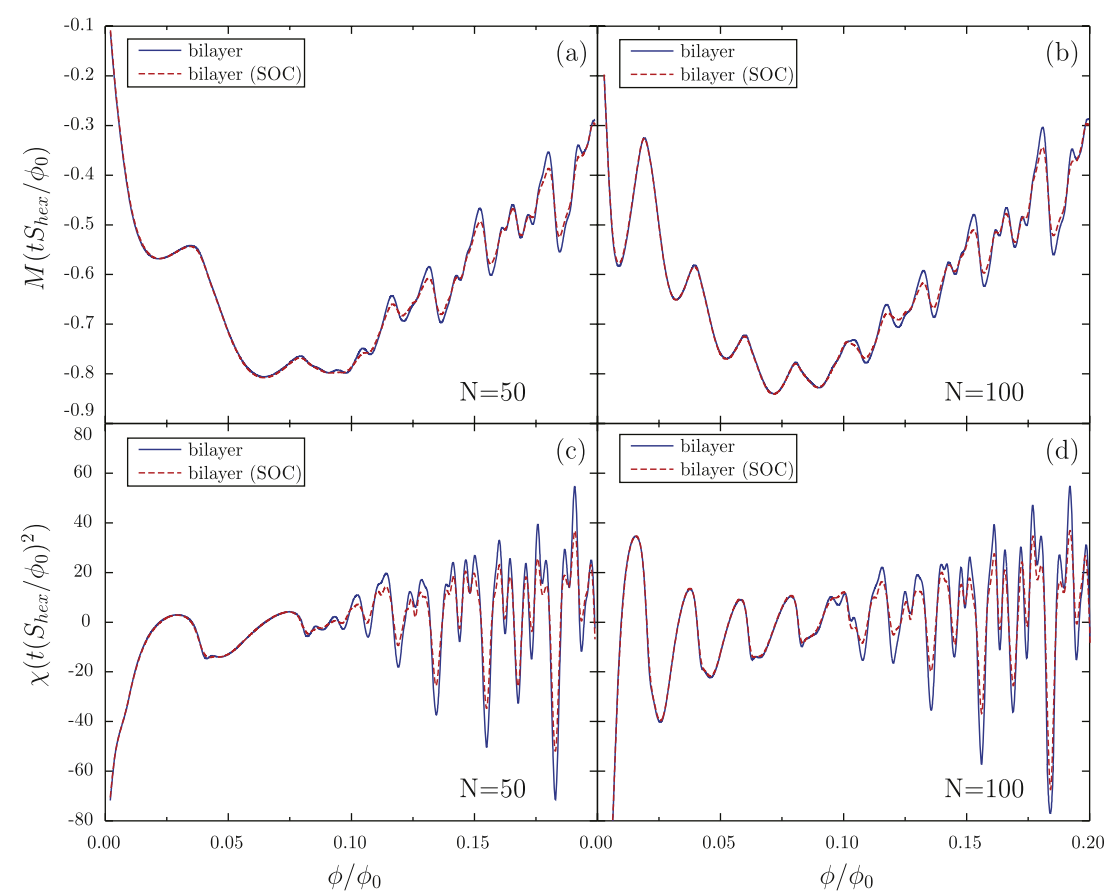

Figure 5. The magnetization and susceptibility for zigzag bilayer ribbons of $N=50$ and $N=100$, chemical potential $=0.0$. The RSO couplings are $\lambda=0$ and 0.1 .

rapidly, reflecting the fast sweeping of the chemical potential through the magnetic subband. The system changes back and forth between the paramagnetic and diamagnetic phases. For graphene nanoribbons, the magnetization contains two distinct oscillations with the magnetic field, the fast oscillation due to the magnetic subband and the slow oscillation due to the size effect (quantization of the transverse momentum). Under a weak magnetic field, the magnetic subbands are not resolved and the large period oscillation is due to the size effect. When the ribbon width is doubled, the oscillation frequency is also doubled. The oscillation due the quantization of the transverse momentum is not affected by the spin-orbit interaction. The fast oscillation due to the magnetic subband is strongly affected by the RSO interaction. The RSO interaction broadens the magnetic subband and reduces the amplitude of the magnetic oscillation. The amplitude reduction is around $50 \%$ for $\lambda=0.1$.

\section{The role of the $\mathrm{RSO}$ interaction on the quantum Hall effect}

The quantum Hall effect in both single layer and bilayer graphene has been well documented and fully analyzed [37-39]. In single layer graphene, the Hall conductance jumps as chemical sweeps through the Landau level. The step (or the size of the jump) is $\sigma_{o}=e^{2} / \hbar$, the same for each jump. As discussed earlier, both the interlayer coupling and the RSO coupling result in level splitting. Under a strong magnetic field, Landau levels are formed in the energy spectrum which lead to discrete peaks in the density of states (DOS). Whenever the Fermi level sweeps over these discrete peaks, the electron concentration jumps and the Hall conductance exhibits a plateau. It is interesting to note that the level at $E=0$ is not affected by either the interlayer coupling or the RSO coupling. In BLG without RSO coupling, the size of the conductance jump at $E=0$ is twice that for subsequent jumps at finite energy. Here we shall show the combined effect of the interlayer coupling and RSO coupling on the quantum Hall effect.

By employing the Green's function method, the Hall conductance is the sum of a bulk term and a surface term, $\sigma^{\mathrm{H}}=\sigma_{x y}^{\mathrm{I}}+\sigma_{x y}^{\mathrm{II}}$, where $\sigma_{x y}^{\mathrm{I}}$ is the bulk term,

$$
\begin{aligned}
\sigma_{x y}^{\mathrm{I}}= & \frac{e^{2}}{2} \mathrm{i} \hbar\left\langle\operatorname { T r } \left[ v_{y} G^{+}\left(E_{\mathrm{F}}\right) v_{x} \delta\left(E_{\mathrm{F}}-H\right)\right.\right. \\
& \left.\left.-v_{y} \delta\left(E_{\mathrm{F}}-H\right) v_{x} G^{-}\left(E_{\mathrm{F}}\right)\right]\right\rangle,
\end{aligned}
$$

and $\sigma_{x y}^{\mathrm{II}}$ is the surface term,

$$
\begin{aligned}
\sigma_{x y}^{\mathrm{II}} & =\frac{e^{2}}{2}\left\langle\operatorname{Tr}\left[\delta\left(E_{\mathrm{F}}-H\right)\left(y v_{x}-x v_{y}\right)\right]\right\rangle \\
& =-e^{2} \operatorname{Tr}\left[\delta\left(E_{\mathrm{F}}-H\right) x v_{y}\right] .
\end{aligned}
$$

The Hall conductance is written as

$$
\begin{aligned}
\sigma^{\mathrm{H}}= & -e^{2} \sum_{n, k}\left\langle\delta\left(E_{\mathrm{F}}-E_{n}(k)\right)\right\rangle\left\langle n, k\left|v_{y}\right| n, k\right\rangle \\
& \times\langle n, k|x| n, k\rangle .
\end{aligned}
$$

Figure 6 depicts the DOS and the Hall conductance in BLG with RSO coupling. There are eight states at $|E|=0$, four for electrons and four for holes. The Hall conductivity depends on the chemical potential i.e., the carrier density. We have shown that there is an equivalence between the interlayer coupling in BLG and the RSO coupling in single layer graphene as both lead to trigonal warping. Here we 


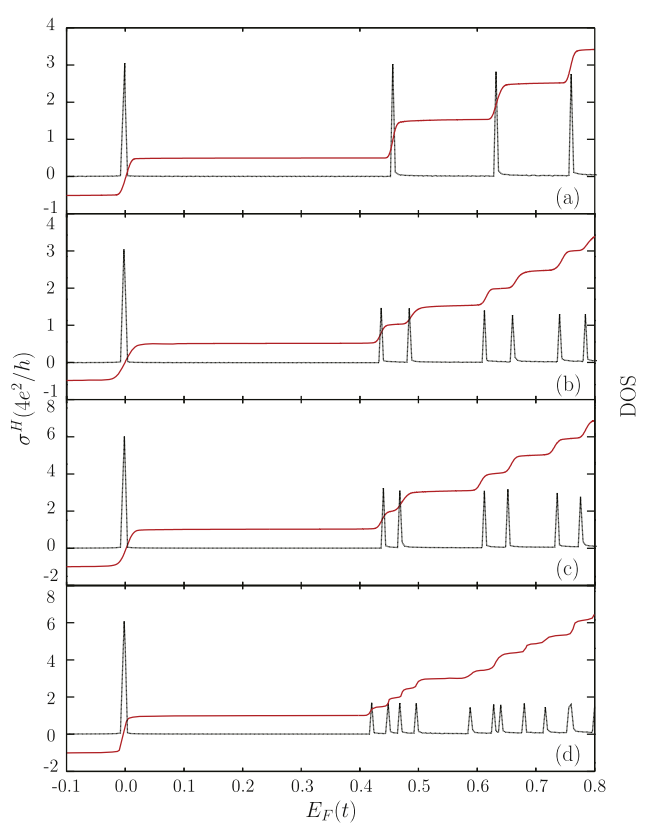

Figure 6. A comparison of the Hall conductances for single layer and bilayer graphene sheets without and with the RSO coupling. (a) single layer graphene, (b) single layer graphene with RSO coupling, (c) bilayer graphene, (d) bilayer graphene with RSO coupling. It should be noted that the height of the plateau is the absolute value but the height of the DOS is a relative value. The height of the DOS is normalized separately according to different parameters.

Therefore the height of the DOS and the height of the plateau are not directly related.

show that the roles played by these two couplings are totally inequivalent in the Hall conductance. The total DOS is conserved when RSO coupling is included. On the other hand, changing from single layer to bilayer doubles the total DOS. There is a simple correlation between the spikes the in difference sub-figures in figure 6. The inclusion of RSO coupling will double the number of spikes (except for the one at $E=0$ ) and the height of the spikes will decrease by a half. On increasing the number of layers from one to two, the spikes at nonzero energy double while their height is unchanged and the spike height at zero energy doubles. Comparing figure 6(a) with figure 6(b), it is found that RSO coupling does not affect the height of the plateau at $E$, but all other plateaus at $E \neq 0$ split into pairs of plateaus of half height. Therefore the overall increase of the Hall conductance in a given energy range is unchanged. The distance between the plateaus within a pair is proportional to the strength of the RSO coupling. On the other hand, comparing figure 6(a) with figure 6(c), it is found that the role of interlayer coupling is to double the height of all plateaus, and then split all plateaus at $E \neq 0$ into pairs of plateaus of half height. The overall effect is that within a fixed energy range, the size of the Hall conductance increase is twice that for single layer graphene (with or without RSO coupling, as shown in figure $6(d)$ ). It is interesting to note that the RSO coupling does not affect the $\epsilon=0$ state due to the coupling requiring finite momentum.

\section{Concluding remarks}

We have studied bilayer graphene in the presence of RSO coupling using a tight-binding model. The properties of its low energy electronic band structure have been investigated. It has been found that the trigonal warpings for the graphite bilayer around the Dirac point are deformed by the presence of RSO coupling. There exist two trigonal warpings with the same central touching point. In the presence of a magnetic field perpendicular to the layers, we have calculated the butterfly spectrum. The changes in the energy spectrum of graphene have been shown as a function of the dimensionless magnetic flux $f=p / q$. The effects of both intra-layer and RSO coupling cause significant distortion to the magnetic subbands. The Landau level is resolved by the RSO interaction in high Landau levels, clearly. Besides breaking the symmetry about $E=0$, the spectra reveal a new magnetic period of $f=3$. The QHE Hall plateaus at the high Landau level $n>0$ have half-integer steps and there is an integer jump at the Landau level $n=0$. We have investigated the splitting that occurs in the DOS of bilayer graphene in the presence of RSO coupling and analyzed the relation to the Hall plateaus.

\section{Acknowledgments}

BW and ZSM acknowledge the financial support from NBRP of China (2012CB921300) and NNSFC Grant (91021017, 11274013). CZ acknowledges support from the Australian Research Council (DP0879115).

\section{Appendix}

Here we show the steps leading to equations (2) and (3) from equation (1). The line index $m$ is defined in figure A.1. The Rashba part of the BLG Hamiltonian is

$$
H_{\mathrm{soc}}=\mathrm{i} \lambda_{\mathrm{R}} \sum_{\langle i, j\rangle} c_{i}^{\dagger}\left(\sigma \times d_{i j}\right)_{z} c_{j} .
$$

For the wavefunctions of sublattices $\mathrm{A}$ and $\mathrm{B}, \psi_{A}$ and $\psi_{B}$, we found

$$
\begin{aligned}
H_{\mathrm{Soc}} \psi_{A}(x)= & \mathrm{i} \lambda_{\mathrm{R}} \mathbf{e}_{z} \cdot\left\{\boldsymbol{\sigma} \times\left[\mathbf{d}(a, 0) \psi_{B}(x-a)\right.\right. \\
& +\mathbf{d}\left(-\frac{a}{2}, \frac{\sqrt{3} a}{2}\right) \mathrm{e}^{\mathrm{i} \frac{2 \pi}{\phi_{0}} \int_{\mathbf{d}} \mathbf{A} \cdot \mathrm{d} \mathbf{l}} \mathrm{e}^{\mathrm{i} k_{y} \frac{\sqrt{3} a}{2}} \\
& \times \psi_{B}\left(x+\frac{a}{2}\right)+\mathbf{d}\left(-\frac{a}{2},-\frac{\sqrt{3} a}{2}\right) \\
& \left.\left.\times \mathrm{e}^{\mathrm{i} \frac{2 \pi}{\phi_{0}} \int_{\mathbf{d}} \mathbf{A} \cdot \mathrm{dl}} \mathrm{e}^{-\mathrm{i} k_{y} \frac{\sqrt{3} a}{2}} \psi_{B}\left(x+\frac{a}{2}\right)\right]\right\} \\
= & \mathrm{i} \lambda_{\mathrm{R}} a \mathbf{e}_{z} \cdot\left\{\mathbf{\sigma} \times\left[\mathbf{d}(1,0) \psi_{B}(x-a)\right.\right. \\
& +\mathbf{d}\left(-\frac{1}{2}, \frac{\sqrt{3}}{2}\right) \mathrm{e}^{\mathrm{i} \frac{2 \pi}{3} f\left(\frac{x}{a}-\frac{1}{4}\right)} \mathrm{e}^{\mathrm{i} k_{y} \frac{\sqrt{3} a}{2}}
\end{aligned}
$$




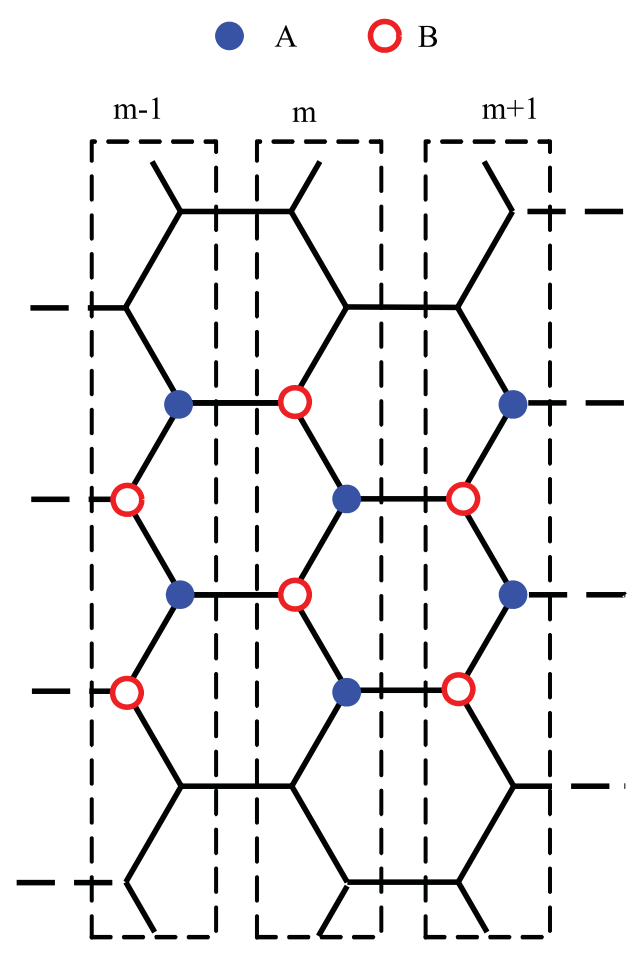

Figure A.1. The definition of the line index of a graphene nanoribbon.

$$
\begin{aligned}
& \times \psi_{B}\left(x+\frac{a}{2}\right)+\mathbf{d}\left(-\frac{1}{2},-\frac{\sqrt{3}}{2}\right) \mathrm{e}^{-\mathrm{i} \frac{2 \pi}{3} f\left(\frac{x}{a}-\frac{1}{4}\right)} \\
& \left.\left.\times \mathrm{e}^{-\mathrm{i} k_{y} \frac{\sqrt{3} a}{2}} \psi_{B}\left(x+\frac{a}{2}\right)\right]\right\} .
\end{aligned}
$$

Taking the notations

$$
\begin{gathered}
\psi_{A_{m}}=\psi_{A}\left(x=\frac{3 a}{2} m\right) \quad \text { and } \\
\psi_{B_{m}}=\psi_{B}\left(x=\frac{3 a}{2} m+\frac{a}{2}\right),
\end{gathered}
$$

we have

$$
\begin{aligned}
H_{\mathrm{soc}} \psi_{A_{m}}= & -\lambda\left[\sigma_{x} \sqrt{3} \sin \left(\pi f\left(m-\frac{1}{6}\right)+\frac{\sqrt{3}}{2} k_{y} a\right)\right. \\
& \left.+\mathrm{i} \sigma_{y} \cos \left(\pi f\left(m-\frac{1}{6}\right)+\frac{\sqrt{3}}{2} k_{y} a\right)\right] \psi_{B_{m}} \\
& -\mathrm{i} \lambda \sigma_{y} \psi_{B_{m+1}}
\end{aligned}
$$

i.e., equation (2). In a similar way, equation (3) can be obtained as follows. We have

$$
\begin{aligned}
H_{\mathrm{soc}} \psi_{B}(x)= & \mathrm{i} \lambda_{\mathrm{R}} \mathbf{e}_{z} \cdot\left\{\boldsymbol{\sigma} \times\left[\mathbf{d}(-a, 0) \psi_{A}(x+a)\right.\right. \\
& +\mathbf{d}\left(\frac{a}{2}, \frac{\sqrt{3} a}{2}\right) \mathrm{e}^{\mathrm{i} \frac{2 \pi}{\phi_{0}} \int_{\mathbf{d}} \mathbf{A} \cdot \mathrm{d} \mathbf{l}} \mathrm{e}^{\mathrm{i} k_{y} \frac{\sqrt{3} a}{2}} \psi_{A}\left(x-\frac{a}{2}\right) \\
& +\mathbf{d}\left(\frac{a}{2},-\frac{\sqrt{3} a}{2}\right) \mathrm{e}^{\mathrm{i} \frac{2 \pi}{\phi_{0}} \int_{\mathbf{d}} \mathbf{A} \cdot \mathrm{d} \mathbf{l}}
\end{aligned}
$$

$$
\begin{aligned}
& \left.\left.\times \mathrm{e}^{-\mathrm{i} k_{y} \frac{\sqrt{3} a}{2}} \psi_{A}\left(x-\frac{a}{2}\right)\right]\right\} \\
= & \mathrm{i} \lambda_{\mathrm{R}} a \mathbf{e}_{z} \cdot\left\{\boldsymbol{\sigma} \times\left[\mathbf{d}(-1,0) \psi_{A}(x+a)\right.\right. \\
& +\mathbf{d}\left(\frac{1}{2}, \frac{\sqrt{3}}{2}\right) \mathrm{e}^{\mathrm{i} \frac{2 \pi}{3} f\left(\frac{x}{a}-\frac{1}{4}\right)} \mathrm{e}^{\mathrm{i} k_{y} \frac{\sqrt{3} a}{2}} \\
& \times \psi_{A}\left(x-\frac{a}{2}\right)+\mathbf{d}\left(\frac{1}{2},-\frac{\sqrt{3}}{2}\right) \\
& \left.\left.\times \mathrm{e}^{-\mathrm{i} \frac{2 \pi}{3} f\left(\frac{x}{a}-\frac{1}{4}\right)} \mathrm{e}^{-\mathrm{i} k_{y} \frac{\sqrt{3} a}{2}} \psi_{A}\left(x-\frac{a}{2}\right)\right]\right\} .
\end{aligned}
$$

With the notations $\psi_{A_{m}}=\psi_{A}\left(x=\frac{3 a}{2} m\right)$ and $\psi_{B_{m}}=$ $\psi_{B}\left(x=\frac{3 a}{2} m+\frac{a}{2}\right)$, we obtain

$$
\begin{aligned}
H_{\mathrm{soc}} \psi_{B_{m}}= & -\lambda\left[\sigma_{x} \sqrt{3} \sin \left(\pi f\left(m+\frac{1}{6}\right)+\frac{\sqrt{3}}{2} k_{y} a\right)\right. \\
& \left.-\mathrm{i} \sigma_{y} \cos \left(\pi f\left(m+\frac{1}{6}\right)+\frac{\sqrt{3}}{2} k_{y} a\right)\right] \psi_{A_{m}} \\
& +\mathrm{i} \lambda \sigma_{y} \psi_{A_{m+1}},
\end{aligned}
$$

i.e., equation (3).

\section{References}

[1] Novoselov K S, Geim A K, Morozov S V, Jiang D, Zhang Y, Dubonos S V, Grigorieva I V and Firsov A A 2004 Science 306666

[2] Geim A K and Novoselov K S 2007 Nature Mater. 6183

[3] Novoselov K S, Geim A K, Morozov S V, Jiang D, Katsnelson M I, Grigorieva I V, Dubonos S V and Firsov A A 2005 Nature 438197

[4] Zhang Y, Tan Y W, Stormer H L and Kim P 2005 Nature 438201

[5] Berger C et al 2006 Science 3121191

[6] Suzuura H and Ando T 2002 Phys. Rev. Lett. 89266603 Suzuura H and Ando T 2003 J. Phys. Soc. Japan 7269

[7] Morozov S V, Novoselov K S, Katsnelson M I, Schedin F, Ponomarenko L A, Jiang D and Geim A K 2006 Phys. Rev. Lett. 97016801

[8] Khveshchenko D V 2006 Phys. Rev. Lett. 97036802

[9] Gusynin V P, Sharapov S G and Carbotte J P 2006 Phys. Rev. Lett. 96256802

[10] Kuzmenko A B, van Heumen E, Carbone F and van der Marel D 2008 Phys. Rev. Lett. 100117401

[11] Nair R R, Blake P, Grigorenko A N, Novoselov K S, Booth T J, Stauber T, Peres N M R and Geim A K 2008 Science 3201308

[12] Liu J, Wright A R, Zhang C and Ma Z 2008 Appl. Phys. Lett. 93041106

[13] Mikhailov S A and Ziegler K 2008 J. Phys.: Condens. Matter 20384204

[14] Wright A R, Xu X G, Cao J C and Zhang C 2009 Appl. Phys. Lett. 95072101

[15] Zhang C and Takahashi Y 1993 J. Phys.: Condens. Matter 55009

[16] Zhang C 1994 Phys. Rev. B 492939

[17] McCann E 2006 Phys. Rev. B 74161403

[18] Ohta T, Bostwick A, Seyller T, Horn K and Rotenberg E 2006 Science 313951 
[19] Abergel D S L and Fal'ko V I 2007 Phys. Rev. B 75155430

[20] Nilsson J and Castro Neto A H 2007 Phys. Rev. Lett. 98126801

[21] Cserti J, Csordas A and David G 2007 Phys. Rev. Lett. 99066802

[22] McClure J W 1957 Phys. Rev. 108612

[23] Slonczewski J C and Weiss P R 1958 Phys. Rev. 109272

[24] Chung D D L 2002 J. Mater. Sci. 371

[25] Nilsson J, Castro Neto A H, Guinea F and Peres N M R 2008 Phys. Rev. B 78045405

[26] Kane C L and Mele M J 2005 Phys. Rev. Lett. 95226801

[27] Min H et al 2006 Phys. Rev. B 74165310

[28] Ertler C et al 2009 Phys. Rev. B 80041405
[29] van Gelderen R and Morais S C 2010 Phys. Rev. B 81125435

[30] Varykhalov A et al 2008 Phys. Rev. Lett. 101157601

[31] Castro Neto A H and Guinea F 2009 Phys. Rev. Lett. 103026804

[32] Zarea M and Sandler N 2009 Phys. Rev. B 79165442

[33] Hasegawa Y and Kohmoto M 2006 Phys. Rev. B 74155415

[34] Mireles F and Schliemann J 2012 arXiv:1203.1094v2

[35] Nemec N and Cuniberti G 2007 Phys. Rev. B 75 201404(R)

[36] Liu J F, Ma Z S, Wright A R and Zhang C 2008 J. Appl. Phys. 103103711

[37] Gusynin V P and Sharapov S G 2005 Phys. Rev. Lett. 95146801

[38] Zhang Y et al 2005 Nature 438201

[39] Abanin D A et al 2007 Phys. Rev. Lett. 98196806 